\title{
WEAK SIMULTANEOUS TRIANGULARIZATION - A DETERMINANT CONDITION
}

\author{
CHEN DUBi
}

Abstract. We introduce and study the notion of weak triangularization for a set of $N$ matrices, which is a weaker version of simultaneous triangularization. We prove that for the special case of 2-by-2 matrices, weak triangularization is equivalent to simultaneous triangularization.

Mathematics subject classification (2000): 15A15.

Key words and phrases: Simultaneous triangularization, weak triangularization, characteristic polynomial, rational transfer function.

\section{REFERENCES}

[1] D. Alpay, C. Dubi: A realization theorem for functions of several complex variables, System and control letters, 49 (2003), 225-229.

[2] I. GOHBERG, P. LANCASTER AND L. RODMAN: Invariant subspaces of matrices with applications, Canadian Mathematical Society Series of Monographs and Advanced Texts, (1986).

[3] H. RadjaVi And P. Rosenthal: Simultaneous triangularization, Springer-Verlag New-York, (2000).

[4] H. RADJAVI: A trace condition equivalent to simultaneous triangularizability; Can. J. Math., Vol. 38, No.2 (1986), 476-386.

[5] C. Dubi: Triangular realization of rational functions of $N$ complex variables, Multidim. Sys. Sign. Process.,to appear.

[6] E. ForNASINI AND G. MARCHESINI: Doubly-indexed dynamical systems: state-space models and structural properties, Math. Systems Theory, 12 (1978/79), 59-72. 